\title{
Avaliação do potencial para a geração de agregados carbono negativos no Brasil
}

As mudanças climáticas são um problema ambiental que causa crescente preocupação na sociedade. Quando se busca solucionar esta questão, diminuir as emissões de $\mathrm{CO} 2$ e outros gases de efeito estufa se apresenta como um grande desafio. Diante disso, o objetivo desse trabalho foi determinar o potencial da produção de agregados para concreto carbono negativos no Brasil, utilizando CO2 capturado de usinas de etanol e escória de siderurgia como matérias primas. Essa avaliação foi conduzida através da determinação do local ideal para instalação deste empreendimento e da estimativa do custo de produção que permite a viabilidade deste produto. Para este fim, foram listadas e mapeadas possíveis fontes de dióxido de carbono e de escória, e operações de geoprocessamento foram aplicadas a esses dados para encontrar o local mais propício para a instalação do empreendimento. 410 usinas de açúcar e etanol e 28 usinas siderúrgicas foram listadas e mapeadas. Devido à grande disponibilidade de matéria prima e a pequena distância entre as fontes, o local considerado mais propício para a instalação desse empreendimento foi Piracicaba no estado de São Paulo. O custo de produção que permite a viabilidade do projeto foi de R\$: 9.742 .924 reais por ano, e apesar desse valor ser baixo, com incentivos governamentais a viabilidade desse empreendimento aumentaria consideravelmente, se tornando uma alternativa para a destinação de escória e uma forma lucrativa de reduzir emissões de dióxido de carbono.

Palavras-chave: Agregados; Etanol; Captura de Carbono.

\section{Evaluation of the potential for the generation of carbon negative aggregates in Brazil}

\begin{abstract}
Climate change is an environmental problem that causes growing concern in society. When addressing this issue, the reduction of $\mathrm{CO} 2$ and other greenhouse gases emissions presents itself as a major challenge. Therefore, the objective of this study was to determine the potential for the production of carbon negative concrete aggregates in Brazil, using $\mathrm{CO} 2$ captured from ethanol plants and steel slag as raw materials. This assessment was conducted by determining the ideal location for installing this enterprise and estimating the production cost that allows the viability of this product. To this end, possible sources of carbon dioxide and slag were mapped, and geoprocessing operations were applied to this data to find the most suitable place for the installation of this project. 410 sugar and ethanol plants and 28 steel mills were listed and mapped. Due to the great availability of raw material and small distance between the sources, Piracicaba in the state of São Paulo was the most favorable place to install this project. The production cost that allows the viability of the project was R\$: 9,742,924 per year, and although this value is low, with government incentives the viability of this venture would increase considerably, becoming an alternative to the disposal of slag and a lucrative way to reduce carbon dioxide emissions.
\end{abstract}

Keywords: Aggregates; Ethanol; Carbon Capture.

Topic: Engenharia Ambiental

Reviewed anonymously in the process of blind peer.
Received: 01/12/2020

Approved: 23/12/2020

Luiza Silva do Nascimento (iD

Instituto Federal Fluminense, Brasil

http://lattes.cnpq.br/0895032354598834

http://orcid.org/0000-0001-5148-5990

luizasnascimento.Isn@gmail.com

Kargean Vianna Barbosa (D)

Instituto Federal Fluminense, Brasil

http://lattes.cnpq.br/5279932752748851

http://orcid.org/0000-0002-8184-607X

kargean@gmail.com

Referencing this:

NASCIMENTO, L. S.; BARBOSA, K. V.. Avaliação do potencial para a geração de agregados carbono negativos no Brasil. Revista Ibero Americana de Ciências Ambientais, v.11, n.7, p.338-348, 2020. DOI: http://doi.org/10.6008/CBPC2179-6858.2020.007.0028 


\section{INTRODUÇÃO}

As mudanças climáticas vêm se destacando como o principal problema ambiental do século XXI, causando grande preocupação às pessoas pelo impacto que terão sobre o planeta e a humanidade. Um dos principais fatores que intensifica as mudanças climáticas é o aumento da concentração dos gases do efeito estufa na atmosfera, sobretudo o dióxido de carbono (ADGER et al., 2009; MOHANTY et al., 2009).

O dióxido de carbono é gerado no processo de respiração aeróbia dos seres vivos heterotróficos e em outros processos naturais, contudo, ele também é o principal subproduto da queima de combustíveis fósseis, da queima de biomassa e diversos processos industriais (BRAGA et al., 2005; IPCC, 2007). Antes do avanço da atividade industrial humana, marcado pela Primeira Revolução Industrial no final do século XVIII, a concentração de $\mathrm{CO}_{2}$ na atmosfera era por volta de 270 ppmv (parte por milhão em volume). Atualmente esse gás tem uma concentração de cerca de 400 ppmv na atmosfera terrestre, e ainda que as emissões de $\mathrm{CO}_{2}$ também possam se originar de uma variedade de fontes naturais, as emissões antropogênicas são responsáveis pelo aumento que ocorreu na atmosfera desde a revolução industrial (UCAR, 2006; US EPA, 2018).

As respostas as mudanças climáticas envolvem duas abordagens: reduzir e estabilizar os níveis de gases de efeito estufa na atmosfera (mitigação) e adaptar-se às mudanças climáticas já em curso. O processo de mitigação consiste em esforços para reduzir ou prevenir a emissão de gases de efeito estufa. A mitigação pode significar o uso de novas tecnologias e energias renováveis, tornando os equipamentos antigos mais eficientes em termos de energia ou alteração das práticas de gerenciamento ou do comportamento do consumidor (UNEP, 2018).

A captura, uso e armazenamento de carbono são técnicas de mitigação que consistem em um processo que captura emissões de dióxido de carbono de fontes como usinas a carvão e as reutiliza ou armazena para que não entrem na atmosfera (US DOE, 2018). A diferença entre a utilização e o armazenamento consiste no destino do $\mathrm{CO}_{2}$ capturado. No armazenamento, o $\mathrm{CO}_{2}$ capturado é transferido para um local adequado para o armazenamento a longo prazo, enquanto que na utilização, o $\mathrm{CO}_{2}$ capturado é convertido em produtos comerciais. A utilização do carbono leva vantagem sobre o armazenamento, pois se apresenta como uma atividade rentável que transforma um passivo em um bem de consumo, trazendo benefícios a longo prazo para o clima (CUÉLLAR-FRANCA et al., 2015; THE GLOBAL CO 2 INITIATIVE, 2018).

A principal aplicação da captura de $\mathrm{CO}_{2}$ são as indústrias de geração de energia, contudo, outras grandes indústrias como as de fabricação de cimento, refino de óleo e gás, produção de amônia, produção de Etanol e fabricação de ferro e aço emitem grandes quantidades de $\mathrm{CO}_{2}$ e podem usar essa tecnologia (IPCC, 2005).

Nos últimos anos registraram-se progressos significativos no uso de $\mathrm{CO}_{2}$ como matéria prima, destacando-se quatro grandes mercados - materiais de construção, intermediários químicos, combustíveis e polímeros ( $\mathrm{CO}_{2}$ SCIENCES \& THE GLOBAL $\mathrm{CO}_{2}$ INITIATIVE, 2016). Cimento e agregados para concreto representam a maior oportunidade a curto prazo para a utilização de $\mathrm{CO}_{2}$, devido ao grande volume destes 
materiais que é comercializado, a permanência da captura do $\mathrm{CO}_{2}$ e a química favorável envolvida em sua produção (SANDALOW et al., 2017).

Além da captura permanente do $\mathrm{CO}_{2}$, a produção de agregados através da captura e uso de carbono tem potencial para converter materiais de baixo valor em produtos úteis, pois utiliza resíduos como matéria prima. Os agregados podem ser utilizados em concreto, asfalto e outras aplicações na construção civil $\left(\mathrm{CO}_{2}\right.$ SCIENCES \& THE GLOBAL CO 2 INITIATIVE, 2016).

A produção dos agregados ocorre através de um processo patenteado chamado de ACT - Tecnologia da Carbonatação Acelerada (HILLS, 1999), que combina $\mathrm{CO}_{2}$ capturado de processos industriais e resíduos térmicos para criar um agregado carbono negativo, pois a quantidade de $\mathrm{CO}_{2}$ capturada excede a quantidade gerada através da fabricação. Para cada tonelada desses agregados produzida, o saldo de emissões de carbono é - $40 \mathrm{~kg}$, ou seja, $40 \mathrm{~kg}$ de $\mathrm{CO}_{2}$ são capturados de forma permanente (CARBON8, 2018).

A Tecnologia da Carbonatação Acelerada consiste em um método de solidificar resíduos. Esse método envolve a adição de aglutinante e, opcionalmente, água aos resíduos, formando uma mistura destes materiais. Simultaneamente durante a formação da mistura e/ou subsequentemente após a formação da mesma, a mistura deve ser tratada com dióxido de carbono suficiente para conseguir a consolidação e o subsequente enrijecimento da referida mistura de modo a produzir um resíduo solidificado (HILLS, 1999).

Diversos materiais e resíduos térmicos podem ser utilizados para a produção de agregados através da tecnologia da carbonatação acelerada, entre eles, a escória de siderurgia. Ao ser tratada pelo processo de ACT para formar agregados para concreto, a escória de siderurgia quando exposta ao $\mathrm{CO}_{2}$ em uma pressão de $300000 \mathrm{~Pa}$ e $12,5 \%$ de seu peso em água, reage com aproximadamente $18 \%$ do seu próprio peso em dióxido de carbono (GUNNING et al., 2014; JOHNSON et al., 2003).

Entre as indústrias que podem ser fontes de $\mathrm{CO}_{2}$ para captura, a indústria de produção de etanol se destaca. A fermentação da cana de açúcar produz um fluxo de $\mathrm{CO}_{2}$ de alta pureza, para cada litro de etanol produzido, geram-se $765 \mathrm{~g}$ de $\mathrm{CO}_{2}$ (IEA, 2011). Outra vantagem é o custo relativamente baixo, em comparação com outras fontes, da captura de $\mathrm{CO}_{2}$ em industrias de etanol, cerca de 20 dólares por tonelada de $\mathrm{CO}_{2}$ capturada (GCCSI, 2017). Diante disso, o processo de fermentação torna-se muito atraente para a captura de Dióxido de Carbono, pois a concentração deste gás é quase $100 \%$ e ele pode então ser capturado diretamente, sem o uso de complexos sistemas de captura (OOST, 2013).

Apesar do alto potencial para diminuir as emissões de $\mathrm{CO}_{2}$, os agregados produzidos através da captura de carbono têm como grande desafio se tornarem competitivos em termos de custos. As principais barreiras para esse produto são os custos que podem ser acarretados por dificuldade de acesso ao $\mathrm{CO}_{2}$ e o transporte dos resíduos que servem de matéria prima ( $\mathrm{CO}_{2}$ SCIENCES \& THE GLOBAL CO 2 INITIATIVE, 2016).

Diante disso, o objetivo desse trabalho foi determinar o potencial da produção de agregados para concreto carbono negativos no Brasil, utilizando $\mathrm{CO}_{2}$ capturado de usinas de etanol e escória de siderurgia como matérias primas, através da determinação do local ideal para este empreendimento e da estimativa do custo de produção que permite a viabilidade deste produto. 


\section{METODOLOGIA}

Para avaliar o potencial da produção de agregados para concreto no Brasil foram seguidas as etapas apresentadas na figura 1.

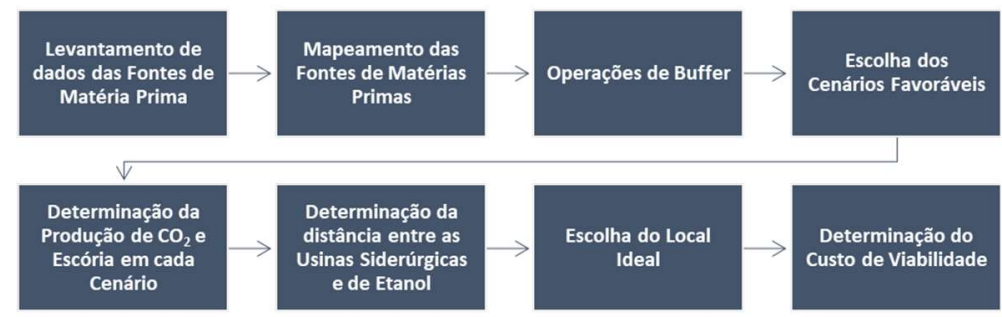

Figura 1: Fluxograma da Metodologia do Trabalho.

Primeiramente, foi realizado o levantamento das fontes de matérias primas: $\mathrm{CO}_{2}$ de usinas de etanol e escória de siderurgia. As usinas de etanol foram escolhidas como fontes de $\mathrm{CO}_{2}$ devido ao baixo custo e simplicidade do processo de captura deste gás. A escória de siderurgia foi escolhida como matéria prima devido à grande quantidade deste resíduo que é gerada no processo de produção de aço, havendo, portanto, grande disponibilidade desses resíduos no Brasil.

Utilizando a plataforma de dados Nova Cana ${ }^{\circledR}$, listaram-se as usinas de açúcar e etanol do Brasil, juntamente com o seu endereço, e status de funcionamento. Essas informações foram colocadas na ferramenta Google Maps ${ }^{\circledR}$ e foi possível obter as coordenadas geográficas de cada uma das usinas e então esses dados foram tabulados.

O mesmo processo foi realizado com as usinas siderúrgicas, de acordo com informações fornecidas pelo Instituto Aço Brasil ( $\left(\mathrm{ABr}^{\circledR}\right)$, as usinas foram listadas e suas coordenadas obtidas através do Google Maps, e esses dados também foram tabulados. O próximo passo foi inserir as tabelas contendo as coordenadas das usinas siderúrgicas e de etanol e açúcar no software $\operatorname{ArcGIS}^{\circledR}$, onde foram gerados shapefiles e as usinas puderam ser mapeadas.

A localização das usinas siderúrgicas foi considerada como base para a instalação do empreendimento para minimizar os custos de transporte, pois a produção de aço, e consequentemente de escória, se dá em grande escala e em poucas fontes, enquanto que a produção de etanol, e do $\mathrm{CO}_{2}$ oriundo da fermentação, é em menor escala e mais difundido pelo país.

A partir da localização das usinas siderúrgicas, foram realizadas operações de geoprocessamento, gerando-se buffers, áreas de influência, de $200 \mathrm{~km}$ (distancia radial) para cada usina siderúrgica.

Com os buffers foi possível avaliar as usinas siderúrgicas que tinham mais usinas de etanol em sua área de influência, e entre elas, foram selecionadas como cenários favoráveis as que tinham mais de 20 usinas de etanol no raio de $200 \mathrm{~km}$, pois a disponibilidade de matéria prima é característica importante na escolha do cenário mais propício para a instalação da produção de agregados.

Para os cenários favoráveis, determinou-se através do Boletim do Etanol $n^{0} 9$ da ANP (Agência Nacional do Petróleo, Gás Natural e Biocombustíveis) (2017) a produção de cada usina de etanol na área de influência. A produção de $\mathrm{CO}_{2}$ proveniente da fermentação foi estimada de acordo com o relatório da IEA 
(2011), onde para cada litro de etanol produzido geram-se $765 \mathrm{~g}$ de $\mathrm{CO}_{2}$.

A produção das usinas siderúrgicas foi obtida no website das empresas, e a produção de escória foi estimada de acordo com Gonçalves (2015), considerando que 360kg de escória são gerados para cada tonelada de aço bruto. A quantidade de $\mathrm{CO}_{2}$ necessária para reagir com a escória de siderurgia pelo processo de ACT foi determinada de acordo com Johnson et al. (2003), considerando necessário $18 \%$ do peso da escória em $\mathrm{CO}_{2}$.

Foi então determinada a distância rodoviária entre cada usina de etanol na área de influência e a usina siderúrgica utilizando o Google Maps ${ }^{\circledR}$. Após a análise da distância entre as fontes de matéria prima, com o objetivo de reduzir os custos relacionados ao transporte, foi então identificado o local mais propício para a instalação do empreendimento em potencial considerando como ideal o cenário que apresentava menor distância entre a fonte de escória e a fonte de $\mathrm{CO}_{2}$.

Para o local escolhido, foi determinado o custo limite que permite a viabilidade do empreendimento. Esse custo foi determinado através do valor de mercado de agregados para concreto convencionais e a margem de lucro de empreendimentos similares, utilizando a fórmula Lucro Bruto = Receitas Totais - Custos Variáveis. Finalmente, foram feitas considerações sobre os desafios que essa tecnologia pode enfrentar e alternativas para superá-los.

\section{RESULTADOS E DISCUSSÃO}

Foram listadas e mapeadas 410 usinas de açúcar e etanol no Brasil, como pode ser observado na Figura 2. Foram também listadas e mapeadas 28 usinas siderúrgicas no país que estão representadas na Figura 3.

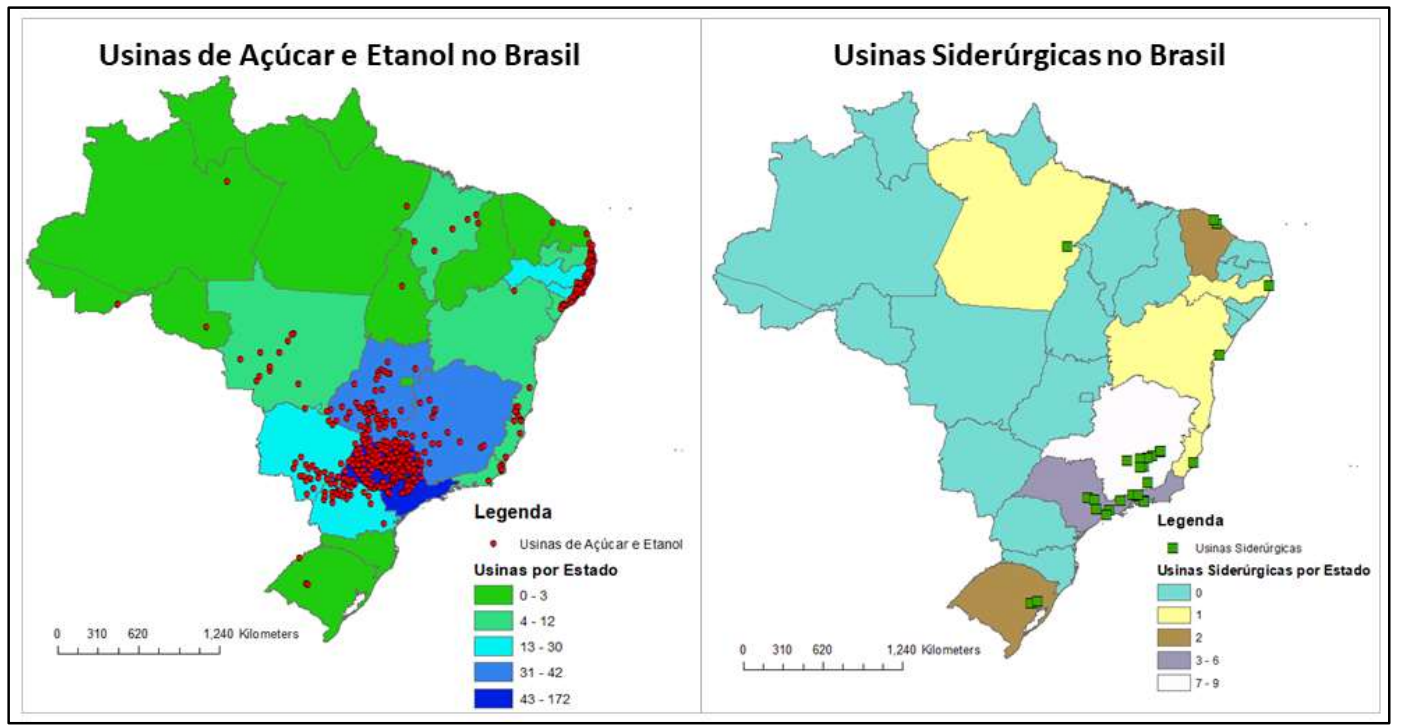

Figura 2: Mapa das Usinas de Açúcar e Etanol do Brasil. Figura 3: Mapa das Usinas Siderúrgicas do Brasil.

Analisando-se a Figura 2 é possível notar que a região sudeste do Brasil se destaca como base da produção de etanol do Brasil, sendo o estado de São Paulo o principal produtor do país, contando com 172 usinas. Para as usinas siderúrgicas, analisando a Figura 3, também se percebe uma presença maior na região sudeste, sendo Minas Gerais, com nove siderúrgicas, o estado com maior número de usinas. 
Ao se comparar a localização das usinas de etanol e das usinas siderúrgicas, utilizando buffers para determinar a área de influência das siderúrgicas, foi possível identificar cenários favoráveis para a implantação do empreendimento devido à presença de matéria prima, sendo esses cenários as usinas siderúrgicas com mais de 20 usinas de etanol ao redor no raio de $200 \mathrm{~km}$.

Dos 28 cenários possíveis apenas 4 tinham mais de 20 usinas de etanol na área do buffer, sendo esses considerados os cenários favoráveis e representados na Figura 4.

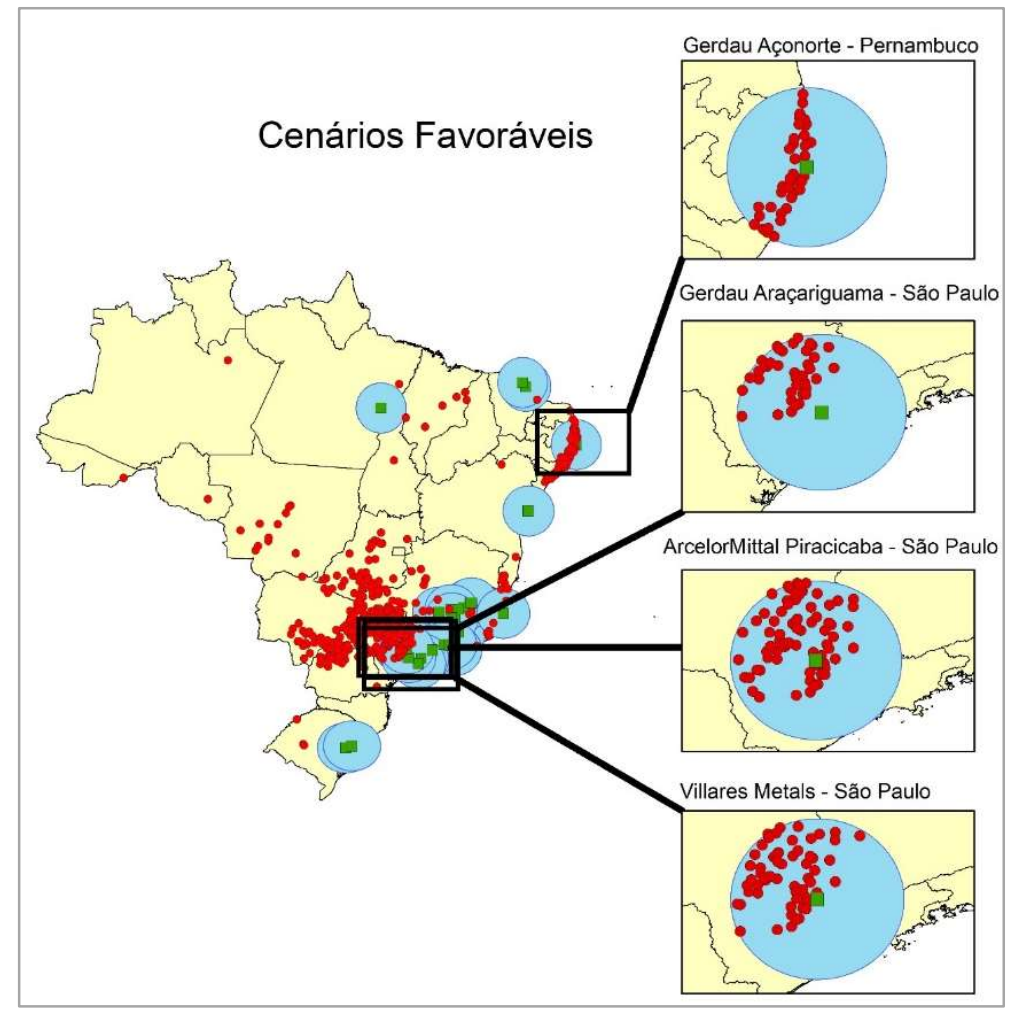

Figura 4: Cenários Favoráveis.

Entre os cenários favoráveis selecionados, um se localiza no estado de Pernambuco e três no estado de São Paulo. O primeiro cenário, Figura 5, Usina Gerdau Açonorte - Pernambuco, conta com 32 usinas de etanol no raio de $200 \mathrm{~km}$.

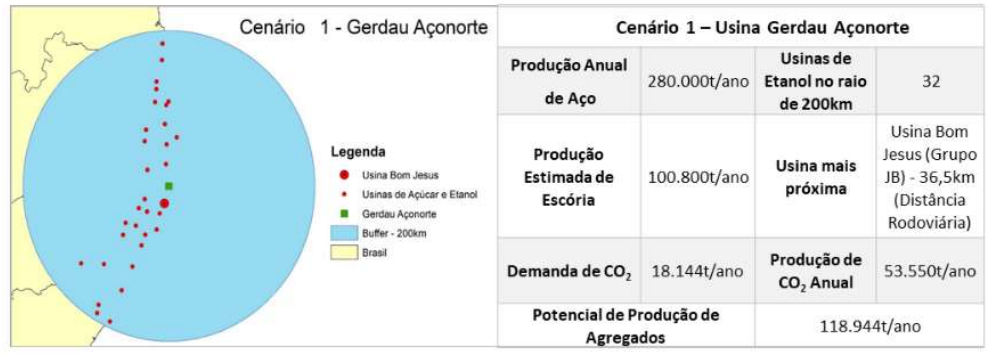

Figura 5: Cenário 1 - Gerdau Açonorte.

Neste cenário, a Figura 5 mostra que a Usina Gerdau Açonorte produz 100.800t/ano de escória. Se toda a escória fosse utilizada para a produção de agregados a demanda de $\mathrm{CO}_{2}$ desse cenário seria de 18.144t/ano e essa demanda poderia ser inteiramente atendida pela produção de $\mathrm{CO}_{2}$ da usina mais próxima, Usina Bom Jesus. No cenário 2, Figura 6, a usina ArcelorMittal Piracicaba - São Paulo, possui 70 usinas de etanol no raio de $200 \mathrm{~km}$. 


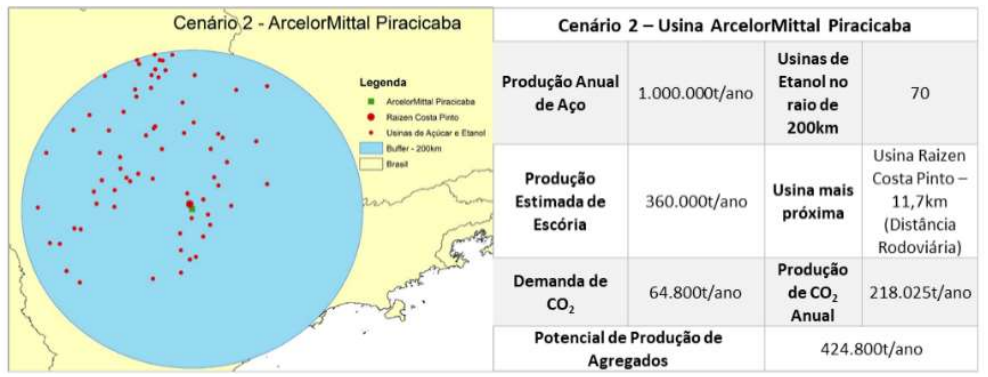

Figura 6: Cenário 2 - ArcelorMittal Piracicaba.

De acordo com a Figura 6, esse cenário tem a usina ArcelorMittal produzindo 360.000t/ano de escória, que se utilizadas para a produção de agregados precisariam de 64.800t/ano de $\mathrm{CO}_{2}$, essa demanda pode ser inteiramente atendida pela usina de etanol mais próxima, Raizen Costa Pinto, que fica a 11,7km de distância. A Figura 7 representa o cenário 3, usina Gerdau Araçariguama - São Paulo, que possui 35 usinas de etanol no raio de $200 \mathrm{~km}$.

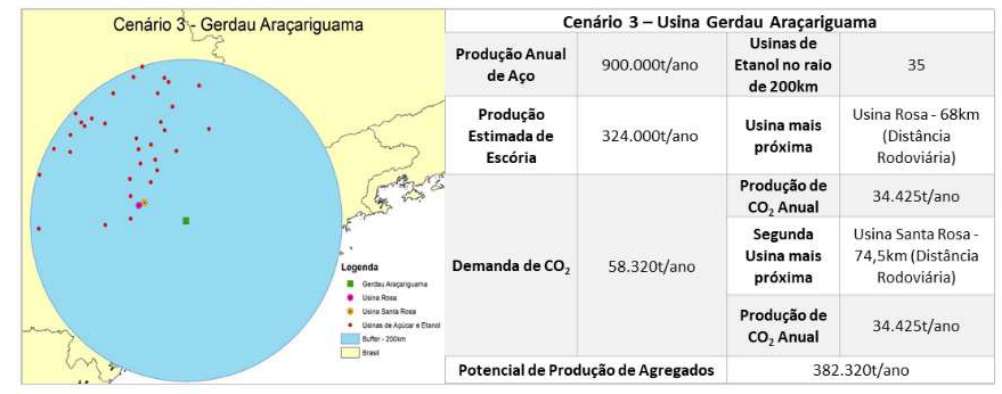

Figura 7: Cenário 3 - Gerdau Araçariguama.

Este cenário, de acordo com a Figura 7, contém a usina Gerdau Araçariguama produzindo 324.000t/ano de escória, que para produção de agregados apresentam a demanda de 58.320t/ano de $\mathrm{CO}_{2}$. Neste cenário a demanda não pode ser atendida pela usina mais próxima, Usina Rosa (68km), necessitando de mais uma usina para que essa demanda seja atendida, Usina Santa Rosa $(74,5 \mathrm{~km})$.

O cenário 4, representado pela figura 8, mostra a Usina Villares Metals - São Paulo, que apresenta 54 usinas de etanol no raio de $200 \mathrm{~km}$.

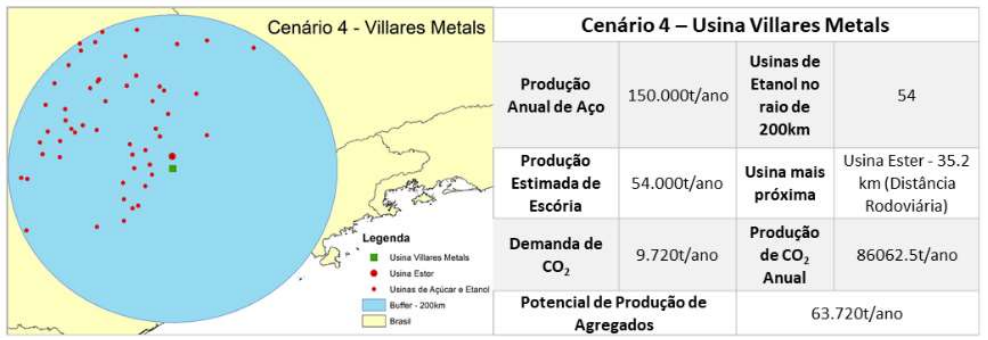

Figura 8: Cenário 4 - Villares Metals.

O último cenário, de acordo com a Figura 8, apresenta a Usina Villares Metals produzindo 54.000t/ano de escória e uma demanda de $9.720 t / a n o$ de $\mathrm{CO}_{2}$ para a produção de agregados. Essa demanda é inteiramente atendida pela Usina Ester, que se localiza a 35,2 km de distância.

Ao se comparar os quatro cenários, o cenário da usina Gerdau Araçariguama se destaca de forma negativa, pois a usina de etanol mais próxima neste cenário dista quase o dobro do que as usinas mais 
próximas nos cenários Gerdau Açonorte e Villares Metals. Outro fator importante é que são necessárias duas usinas de etanol para produzir $\mathrm{CO}_{2}$ suficiente para este cenário se toda a escória fosse ser convertida em agregados, enquanto que nos outros cenários a usina mais próxima atendeu as demandas por inteiro.

Em relação aos cenários Gerdau Açonorte, ArcelorMittal Piracicaba e Villares Metals, o cenário ArcelorMittal Piracicaba se destaca por ter a menor distância entre a usina siderúrgica e a usina de etanol mais próxima e a maior quantidade de usinas de etanol no raio de $200 \mathrm{~km}$.

De acordo com as organizações $\mathrm{CO}_{2}$ Sciences \& The Global $\mathrm{CO}_{2}$ Initiative (2016) a dificuldade de acesso ao $\mathrm{CO}_{2}$ e os custos de transportes das matérias primas são as principais barreiras para que agregados produzidos através de Captura de carbono sejam competitivos no mercado. Diante disso, o cenário mais propício para a instalação do empreendimento para produzir agregados para concreto através da captura de carbono de usinas de etanol e escória de usinas siderúrgicas é o cenário da usina ArcelorMittal Piracicaba.

Aliada a grande disponibilidade de matérias primas para a produção dos agregados, a localização do cenário 2 é muito interessante para a instalação desse empreendimento devido à pouca distância de Piracicaba para a capital, São Paulo $(155 \mathrm{~km})$, e ao grande consumo de agregados do estado de São Paulo como um todo, 4,5 t/hab/ano (VALVERDE, 2011), o que pode auxiliar na absorção dos agregados pelo mercado.

Para o cenário escolhido, simulou-se a instalação de um empreendimento ideal que produziria a quantidade total de agregados permitida pelo cenário. Sabe-se que a escória de siderurgia já é reciclada em outros processos, e, portanto, o uso integral da escória produzida no cenário pode ser dificultoso, contudo, para efeito de simulação dos custos, assumiu-se que os agregados seriam produzidos de acordo com o potencial total do cenário. No Cenário da ArcelorMittal Piracicaba essa produção máxima é de 424.800t/ano.

A partir da produção do cenário foi estimado o custo limite que permite a viabilidade do empreendimento. Esse custo foi obtido ao simular que o empreendimento em Piracicaba obteria a mesma margem de lucro que o empreendimento Carbon8, instalado na Inglaterra, que produz agregados carbono negativos utilizando o processo de ACT, e tem como lucro bruto anual 1,4 milhões de dólares referentes a uma produção de 150.000 toneladas de agregados por ano, ou seja, 9,3 dólares de lucro bruto por tonelada de agregado produzida (CARBON8, 2017). Foi necessário fazer essa simulação, pois não foi possível averiguar o custo real do transporte junto a transportadoras que realizam esse serviço, e a tecnologia de produção de agregados com resíduos térmicos e $\mathrm{CO}_{2}$ é relativamente nova, e, portanto, não foi possível determinar os custos de produção desse material.

Assumindo-se que o valor de venda dos agregados produzidos seria o mesmo que dos agregados convencionais no Brasil para facilitar a absorção dos mesmos pelo mercado, foi possível determinar a receita, e subtraindo-se o lucro bruto da receita foi possível obter o valor limite dos custos variáveis que permitiria a viabilidade desse empreendimento (Tabela 1 ).

O custo limite de viabilidade obtido, 9.742.924 reais por ano, representa um valor limite para o custo de produção que possibilita a viabilidade do projeto. Esses custos compreenderiam as despesas com transporte, energia e operação do empreendimento em si. 
Tabela 1: Custo Limite de Viabilidade.

\begin{tabular}{|c|c|}
\hline \multicolumn{2}{|c|}{ Análise do Custo Limite de Viabilidade } \\
\hline Produção Carbon8 & $150.000 t /$ ano \\
\hline Lucro Bruto & 1.400 .000 milhões de dólares/ano \\
\hline Produção Cenário Piracicaba & $424.800 t /$ ano \\
\hline Lucro Bruto Proporcional & $\begin{array}{c}3.964 .800 \text { milhões de dólares por ano } \\
\text { ou } 16.499 .912 \text { milhões de reais } \\
\text { (Cotação 15/01/2020) }\end{array}$ \\
\hline $\begin{array}{c}\text { Receita da venda de 424.800t de } \\
\text { agregados ao preço de agregados } \\
\text { convencionais - 61,77 reais/tonelada } \\
\text { CONSTRUDATA, 2018) }\end{array}$ & 26.242 .837 reais por ano. \\
\hline Custos & \\
\hline
\end{tabular}

O custo limite de viabilidade obtido é relativamente baixo, e provavelmente não seria suficiente para cobrir as despesas relativas à produção desse produto, porém um fator importante a se considerar é o valor de venda desses agregados. Para efeito de estimativa de custos para a viabilidade foi adotado o valor comercial de agregados convencionais, contudo, o valor de venda desses materiais poderia ser superior. Os agregados produzidos através de captura de carbono têm um apelo comercial de sustentabilidade grande, e pela produção em pequena escala quando comparada a produção anual de agregados convencionais no Brasil eles devem ser facilmente absorvidos pelo mercado de construção verde e por empreendimentos que busquem certificações ambientais como a LEED (Liderança em Energia e Design Ambiental).

$\mathrm{O}$ impacto que a produção desses agregados tem sobre as emissões de $\mathrm{CO}_{2}$ é um fator importante a ser analisado. Segundo a organização Anepac (Associação Nacional das Entidades de Produtores de Agregados para Construção), em 2014 foram produzidas no Brasil 741 milhões de toneladas de agregados para concreto, sendo 439 milhões de toneladas de areia e 302 milhões de toneladas de brita (2014). Se 10\% da produção de brita fosse substituída por agregados carbono negativos, seriam produzidos 30,2 milhões de toneladas desses agregados e 1.2 milhões de toneladas de $\mathrm{CO}_{2}$ seriam permanentemente capturados anualmente.

Subsídios governamentais, para manter os preços acessíveis e incentivar o consumo desse material, e corte de impostos para empreendimentos e construções que usem materiais sustentáveis são alternativas que podem aumentar a chance comercial dos agregados de dióxido de carbono. Entre as vantagens da produção desse material pode-se listar a redução de emissões de $\mathrm{CO}_{2}$, utilização de resíduos como matéria prima e a capacidade de ser uma alternativa para locais onde as pedreiras tiveram sua capacidade de produção esgotada.

\section{CONCLUSÕES}

Foi possível identificar quatro cenários favoráveis que, combinados, tem a capacidade de produzir 989.784t/ano de agregados gerados a partir de escória de siderurgia e $\mathrm{CO}_{2}$ capturado de usinas de etanol.

Entre os cenários favoráveis, o cenário 2 - ArcelorMittal Piracicaba foi identificado como o local ideal para a instalação da produção de agregados, tendo o maior número de usinas de etanol em sua área de influência e a menor distância entre a siderúrgica e a usina de etanol. Esse cenário tem o potencial de produzir 
até 424.800 t/ano de agregados e capturar permanentemente 16,9 toneladas de $\mathrm{CO}_{2}$ por ano.

O custo limite de viabilidade do empreendimento foi de $R \$$ : 9.742 .924 reais por ano, e apesar desse valor indicar que esses agregados podem não ser competitivos em relação ao seu custo, aumentando-se o valor de venda desses agregados e com incentivos governamentais, a viabilidade desse empreendimento aumenta consideravelmente, se tornando uma alternativa para a destinação de escória e uma forma lucrativa de reduzir emissões de dióxido de carbono.

AGRADECIMENTOS: À Coordenação de Aperfeiçoamento de Pessoal de Nível Superior - CAPES, à Stony Brook University e ao Instituto Federal Fluminense.

\section{REFERÊNCIAS}

ADGER, W. N.; BARNETT, J.. Four reasons for concern about adaptation to climate change. Environment and Planning A, v.41, n.12, p.2800-2805, 2009. DOI:

https://doi.org/10.1068/a42244

ANEPAC. Associação Nacional das Entidades de Produtores de Agregados para Construção. Mercado: Perspectivas para o Setor de Agregados. São Paulo: ANEPAC, 2014.

ANP. Agência Nacional do Petróleo, Gás Natural e Biocombustíveis. Boletim do Etanol n. 09/2017. Brasília: ANP, 2017.

BRAGA, B.; HESPANHOL, I.; CONEJO, G. L. J.; MIERZWA, C. J.; DE BARROS, T. L. M.; SPENCER, M.; PORTO, M.; NUCCI, N.; JULIANO, N.; EIGER, S.. Introdução à Engenharia Ambiental: $O$ desafio do desenvolvimento sustentável. 2 ed. Pearson Prentice Hall, 2005.

CARBON8. Carbon8 Aggregates: Technical Data Sheet. Kent: Carbon8, 2018

CARBON8. Carbon8 Systems: Developing an innovative and profitable process that combines waste $\mathrm{CO}_{2}$ and thermal residues, to create a carbon negative aggregate for construction and lock $\mathrm{CO}_{2}$ in for good within our built environment. Kent: Carbon8, 2017.

$\mathrm{CO}_{2}$ SCIENCES \& THE GLOBAL CO 2 INITIATIVE. Global Roadmap for Implementing $\mathrm{CO}_{2}$ Utilization. Ann Arbor: University of Michigan, 2016.

CONSTRUDATA. SindusCon SP: Preços de Materiais: Brita 2. São Paulo: Construdata, 2018.

CUÉLLAR-FRANCA, R. M.; AZAPAGIC, A.. Carbon capture, storage and utilization technologies: A critical analysis and comparison of their life cycle environmental impacts. Journal of $\mathrm{CO}_{2}$ Utilization, v.9, p.82-102, 2015. DOI: https://doi.org/10.1016/j.jcou.2014.12.001

GCCSI. Global costs of carbon capture and storage: 2017 Update. Melbourne, Australia: Global CCS Institute, 2017.

GONÇALVES, D. R. R.. Análise da viabilidade econômica via simulação de Monte Carlo para utilização da escória de aciaria como agregado na fabricação de pré-fabricados para a construção civil: ecoblocos. Dissertação (Mestrado em Engenharia Civil) - Universidade Federal de Ouro Preto, Ouro Preto, 2015.

GUNNING, P.; HILLS, C.. Carbon negative: First commercial application of accelerated carbonation technology. In: INTERNATIONAL SCIENTIFIC CONFERENCE: SCIENCE AND HIGHER EDUCATION IN FUNCTION OF SUSTAINABLE DEVELOPMENT, 7. Anais. Sérvia: Uzice, 2014.

HILLS, C.. Hazardous waste treatment. U.S. Patent, 1999.

IEA. Potential for biomass and carbon dioxide capture and storage: IEA Greenhouse Gas R\&D Programme. Cheltenham: Ecofys, 2011.

IPCC. Intergovernmental Panel on Climate Change. Climate change 2007: The physical science basis. The Intergovernmental Panel on Climate Change. Agenda, v.6, n.07, p.333, 2007

IPCC. Intergovernmental Panel on Climate Change. IPCC Special Report on Carbon Dioxide Capture and Storage. The Intergovernmental Panel on Climate Change. Cambridge: Cambridge University Press, United Kingdom and New York, 2005.

JOHNSON, D. C.; MACLEOD, C. L.; CAREY, P. J.; HILLS, C. D. Solidification of stainless-steel slag by accelerated carbonation. Environmental Technology, v.24, n.6, p.671678, 2003. DOI: https://doi.org/10.1080/09593330309385602

MOHANTY, S.; MOHANTY, B. P.. Global climate change: a cause of concern. National Academy Science Letters, v.32, n.5-6, p.149-156, 2009.

OOST, M. C.. Assessment of the techno-economic feasibility of bioethanol production with carbon capture and storage in Brazil. Dissertation (Master in Energy Science) - Utrecht University, Utrecht, 2013.

SANDALOW, D.; AINES, R.; FRIEDMANN, J.; MCCORMICK, C.; MCCOY, S.. Carbon dioxide utilization (CO2U)-ICEF roadmap 2.0. Prepared to facilitate dialogue at the Fourth Innovation on for Cool Earth Forum. Lawrence Livermore National Laboratory Report, TR739322. 2017. 
THE GLOBAL $\mathrm{CO}_{2}$ INITIATIVE. Transforming $\mathrm{CO}_{2}$ : Carbonnegative, dollar-positive. Ann Arbor: University of Michigan, 2018.

UCAR. University Corporation for Atmospheric Research. Carbon Dioxide. Boulder: University Corporation for Atmospheric Research, 2006.

UNEP. United Nations Environment Programme. Mitigation. Nairobi: United Nations Environment Programme, 2018.
US DOE. Department of Energy: Carbon Capture, Utilization \& Storage. Washington: US DOE, 2018.

US EPA. Greenhouse Gas Emissions: Overview of Greenhouse Gases. Washington: US EPA, 2018.

VALVERDE, F. M.. Agregados para construção civil. Balanço mineral brasileiro, 2011.

A CBPC - Companhia Brasileira de Produção Científica (CNPJ: 11.221.422/0001-03) detém os direitos materiais desta publicação. Os direitos referem-se à publicação do trabalho em qualquer parte do mundo, incluindo os direitos às renovações, expansões e disseminações da contribuição, bem como outros direitos subsidiários. Todos os trabalhos publicados eletronicamente poderão posteriormente ser publicados em coletâneas impressas sob coordenação da Sustenere Publishing, da Companhia Brasileira de Produção Científica e seus parceiros autorizados. Os (as) autores (as) preservam os direitos autorais, mas não têm permissão para a publicação da contribuição em outro meio, impresso ou digital, em português ou em tradução. 